\section{A RETROSPECTIVE CASE SERIES ON THE EFFECTIVENESS AND SAFETY OF A NOVEL ULTRASOUND-GUIDED CLAVIPECTORAL PLANE BLOCK FOR MIDSHAFT CLAVICLE SURGERIES}

A Rosales*, N Aypa. Makati Medical Center, Makati, Philippines

\subsection{6/rapm-2021-ESRA. 160}

Background and Aims The use of brachial plexus block (interscalene approach) or combination blocks (interscalene with superficial cervical plexus) in clavicle surgeries can be time consuming versus using a clavipectoral plane block (CPB), a novel block first described in the 2017 European Society of Regional Anaesthesia Congress. The primary objective of this study was to demonstrate the effectiveness and safety of using $\mathrm{CPB}$ with or without superficial cervical plexus (SCP) block as surgical anesthesia in clavicle surgeries.

Methods This was a retrospective, descriptive, observational case series of seven patients with midshaft clavicle fractures approved by the IRB (Protocol Number 2021-047). The primary endpoint was to determine the pain scores and opioid consumption of the patients thru a chart review and to determine any adverse effects of the blocks.

Results Both patients given with CPB had pain after PACU discharge up to 24 hours after surgery, but none required opioid medications post-op. All five patients who had CPB with SCP block had pain after PACU discharge up to 24 hours after surgery but one patient previously under sedation had required opioid medication after PACU discharge. The median duration between PACU discharge and first post-operative pain reported was at 12 hours. None of the patients had any adverse effects from the nerve blocks received.

Conclusions CPB with or without SCP block provided effective and safe anesthesia and analgesia to patients who underwent clavicle surgery under general anesthesia or intravenous

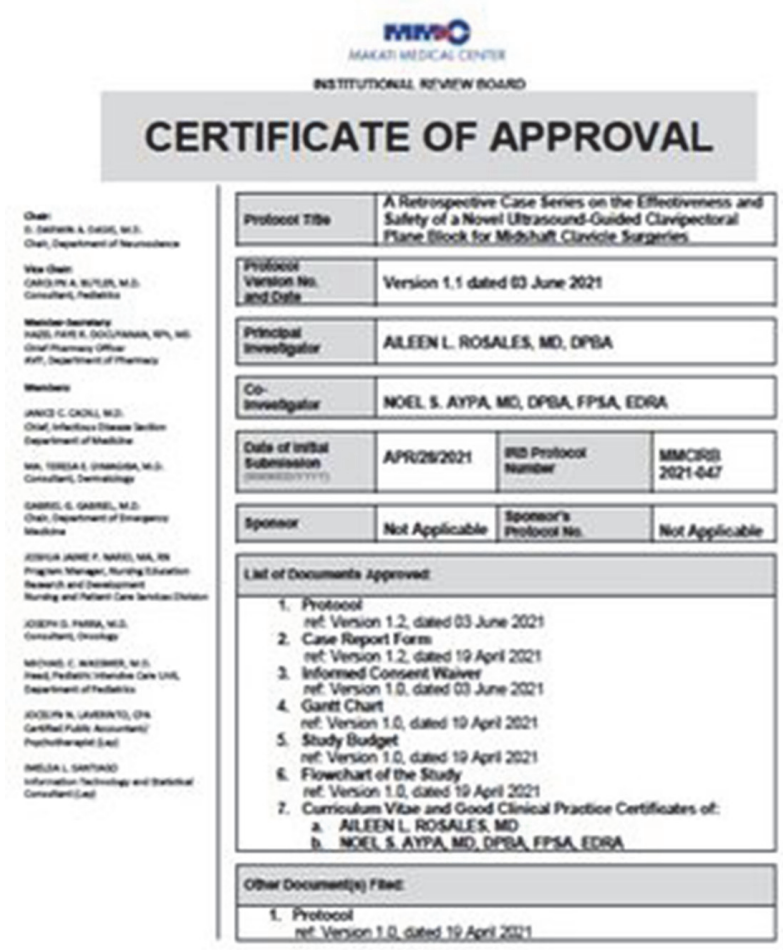

Abstract 160 Figure 1

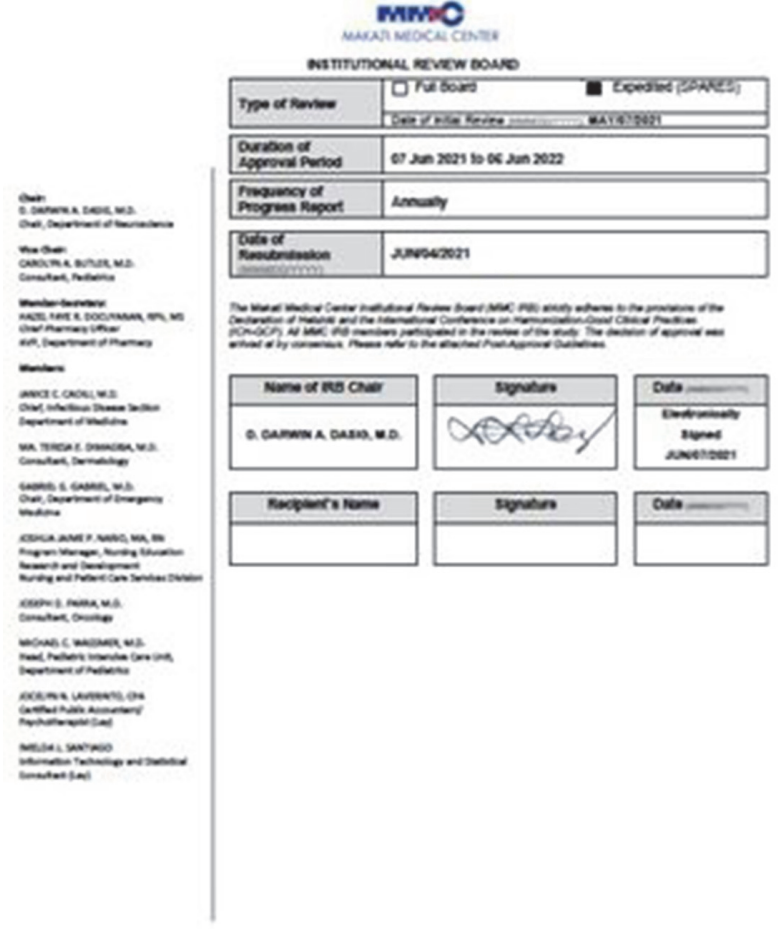

Abstract 160 Figure 2

sedation. However, larger prospective trial studies should be conducted to further illustrate the sensory distribution of this plane block.

\section{INCIDENCE OF PNEUMOTHORAX FROM CHEST WALL BLOCKS FOR AMBULATORY BREAST CANCER SURGERY IN A HIGH-VOLUME PRACTICE}

H Tokita*. Memorial Sloan Kettering Cancer Center, New York, USA

\subsection{6/rapm-2021-ESRA.161}

Background and Aims There is growing interest in regional anesthesia for breast surgery however many clinicians avoid paravertebral blocks (PVB) for fear of causing pneumothorax due to proximity to the pleura. Fascial plane blocks have been proposed as alternative approaches that may be safer for patients. We describe the rate of pneumothorax from PVB and serratus blocks in our high-volume practice.

Methods This IRB-approved retrospective study assessed 2,793 patients who received a regional block for mastectomy from January 5, 2016 - April 22, 2020 subdivided by laterality and block type (Table 1). We calculated risk of pneumothorax (including 95\% C.I.) for patients who received: at least one PVB; at least one serratus block; unilateral blocks; and bilateral blocks. All blocks were placed preoperatively with ultrasound-guidance by an experienced anesthesiologist or supervised trainee.

Results PVB: 2251 patients, one pneumothorax. Complication rate $0.04 \%$; $95 \%$ C.I. $<0.01 \%, 0.2 \%$

Serratus: 520 patients, one pneumothorax. Complication rate $0.2 \% ; 95 \%$ C.I. $<0.01 \%, 1.1 \%$

Unilateral: 1081 patients, no pneumothorax. Complication rate $0 \% ; 95 \%$ C.I. $0 \%, 0.3 \%$ 
Bilateral: 1712 patients, two pneumothoraces. Complication rate $0.1 \% ; 95 \%$ C.I. $<0.01 \%, 0.4 \%$

Conclusions Pneumothorax is a rare complication of ultrasound-guided PVB and serratus blocks in a high-volume practice. This aligns with the prior findings of $\operatorname{Pace}^{1}$ in a retrospective study of 856 patients who received ultrasoundguided PVB, none of whom had suspected pneumothorax. We report a slightly higher rate of pneumothorax with serratus blocks, suggesting that fascial plane blocks are not necessarily 'safer.'

\section{ULTRASOUND GUIDED SUPRACLAVICULAR BRACHIAL PLEXUS BLOCK WITH 0.5\% BUPIVACAINE AND ADDITIVES: CASE SERIES AT TEACHING HOSPITAL ANURADHAPURA}

M Hapugoda*. Teaching Hospital Anuradhapura, Anuradhapura, Sri Lanka

\subsection{6/rapm-2021-ESRA. 162}

Background and Aims Supraclavicular block (SCB) is associated with excellent post-operative patient outcomes for upper limb surgeries. Bupivacaine, a long-acting regional anaesthetic, efficacy of which is altered with the co-administration of additives.

Aim of the study was to assess the efficacy of supraclavicular block with $0.5 \%$ bupivacaine compared to co-administration of additives and the associated complications.

Methods Following ethical clearance and informed consent, over a period of 5 months from June 2020, 152 adult patients at Teaching Hospital Anuradhapura Sri Lanka, undergoing upper limb surgeries were divided into 4 groups \& prospectively followed-up. All received $0.5 \%$ of Bupivacaine while additives 2\% Lidocaine, $8.4 \%$ sodium bicarbonate \& $8 \mathrm{mg}$ Dexamethasone was added to other 3 groups. Sensory and Motor block onset time, duration of post-block analgesia, acute and late complications and patient satisfaction was noted.Data was analysed using descriptive statistics \& ANOVA, using SPSS V.25.

Results Successful surgical anesthesia was achieved in all with 0 cases of long-term neurological complications with 94\% patient satisfaction. The motor \& sensory block onset time \& post block analgesia duration respectively for Lidocaine (9.74min,9.74 $\mathrm{min} \& 7.07 \mathrm{~h})$, Bicarbonate $(12.89 \mathrm{~min}$, $16.32 \mathrm{~min} \& 12.09 \mathrm{~h}$ ), dexamethasone (19. $34 \mathrm{~min}, 17.24 \mathrm{~min}$ $\&$ 20.87h) \& Bupivacaine was (20.39min, 18.42min \& 13.15h).

Conclusions The differences between bupivacaine and lidocaine groups for sensory \& motor block onset times \& between Bupivacaine \& dexamethasone groups for post-block analgesia duration were statistically significant $(\mathrm{p}<.001)$. Supraclavicular block has minimal associated complications \& additives Lidocaine shortens the onset of anaesthesia and the duration of analgesia while dexamethasone prolongs the duration of analgesia significantly.

\section{AN OPEN CONVERTED ABDOMINAL SURGERY IN ERAS (ENHANCED RECOVERY AFTER SURGERY) PROTOCOL}

C Marchandise*, G Samouri, N Parisi, P Creyf. Saint-Pierre, Ottignies, Belgium
Abstract 163 Table 1

\begin{tabular}{|r|r|r|}
\hline & Patient 1 & Patient 2 \\
\hline Drink water & $\mathrm{H} 0$ & $\mathrm{H} 2$ \\
\hline Eat a rusk & $\mathrm{H} 6$ & $\mathrm{H} 6$ \\
\hline Stand up \& toilet & $\mathrm{H} 6$ & $\mathrm{H} 6$ \\
\hline Intestinal transit & $\mathrm{H} 36$ & $\mathrm{H} 40$ \\
\hline Catheters out & $\mathrm{H} 36$ & $\mathrm{H} 42$ \\
\hline Discharge & $\mathrm{H} 48$ & $\mathrm{H} 96$ \\
\hline
\end{tabular}

Background and Aims Described by Forero et al, the Erector Spinae Plane Block (ESPB) is a multidermatomal sensory block that provides regional anesthesia to the ipsilateral thoracic or abdominal wall.

We report two cases of Enhanced Recovery laparoscopic after sigmoidectomy converted to laparotomy, combining multimodal analgesia with bilateral ESPB for postoperative analgesia.

Methods The plan was general anesthesia with minimal stress surgery care; using opioid free anaesthesia, dexmedetomidine, dexamethasone and NSAID. Early in the procedure, the surgeon converted to open surgery so we decided to keep the ERAS protocol with a bilateral ESPB which would ensure more comfort.

The ultrasound-guided injection of a long acting local anaesthetic between the erector spinae muscle and the transverse spinal process is followed by the placement of a catheter for continuous infusion (L-bupivacaine $0.125 \%$ at the rate of $6 \mathrm{ml} / \mathrm{h})$.

Results The ERAS protocol was ensured. The Numerical Rating Scale scores were always below 3/10 except on day 2, at the withdrawal of the catheter relieved by $5 \mathrm{mg}$ of oxycodone. No nausea or vomiting were reported. (Table 1)

Conclusions Bilateral ESPB is a safe technique with no major side effects compared to the epidural. It gives good quality analgesia and it provides a faster recovery with early standing so a good option in converted abdominal surgery. Prospective randomized trials are needed to confirm the apply of this block.

\section{ANAESTHESIA AND POSTOPERATIVE ANALGESIA FOR FOREFOOT SURGERY - A REVIEW OF OUR CURRENT PRACTICE AT NOTTINGHAM CITY HOSPITAL}

${ }^{1} \mathrm{~N}$ Sadavarte ${ }^{*},{ }^{2} \mathrm{~T}$ Chen, ${ }^{1} \mathrm{~V}$ Thanawala. ${ }^{1}$ Nottingham University Hospitals NHS Trust, Nottingham, UK; ${ }^{2}$ University of Nottingham Medical School, Nottingham, UK

\subsection{6/rapm-2021-ESRA.164}

Background and Aims Foot and ankle surgery are associated with moderate to severe pain which can influence the postoperative outcome. We performed an audit to review our practice for forefoot surgery at Nottingham City Hospital based 